ment have been developed and these have been essential for the expansion of renal. liver. and heart transplantation. Currently liver grafts are usually harvested as part of a multiple organ procurement. which can be performed easily in brain dead donors without jeopardizing any of the organs. ${ }^{9}$ Combined procurements involving harvesting of the liver include the following organ combinations in order of frequency: liver and kidneys; liver. kidney's and heart: and liver, kidneys and heart/lung. The principles responsible for the successful removal of multiple combinations of organs with maintenance of the integrity of each organ. along with its blood supply and its ability to function reliably after transplantation, are dependent upon the application of methods for the rapid core cooling of the solid organs by aortic infusion of cold electrolute- or colloid-containing solutions. In the case of the liver. further and more rapid core cooling is achieved by additional infusion of cold solution through a cannula placed in the portal venous system.

Detailed descriptions of the methods and technical details of multiple organ retrieval developed at the University of Pittsburgh and adopted as standard by most other groups have been published. $9 . "$ There are two basic techniques of liver harvesting as part of a multiple organ retrieval known as the "standard" or traditional and the "rapid" technique. Both techniques have major advantages, and under different circumstances. can be combined to different degrees. Both can be used when working with other surgical retrieval teams, and will result in good quality organs.

For both techniques excellent exposure is achieved through a complete midline sternal-splitting and abdominal incision. The "standard" technique will be described first. Division of the left triangular ligament allows mobilization of the left lateral segment of the liver, and the supraceliac aorta below the diaphragm to be encircled. The hilar structures of the liver are then dissected free; the common bile duct is divided far distally, the portal and splenic veins are identified, and care is taken to manipulate the arterial supply as little as possible.

Variations or anomalies in the hepatic arterial supply complicate the hilar dissection in up to one third of donors: any or all of the liver may be supplied by branches of the left gastric artery in the gastrohepatic ligament. superior mesenteric artery, or direct branches from the aorta. Various methods have been described to reconstruct these complex donor anomalies and multiple arteries, which allow all such livers to be used... :

Once this preliminary dissection is completed, cannulae are inserted into the distal abdominal aorta and splenic vein (portal system) for the infusions. Thus the procedure can be terminated at any time, and the specific organs that are to be removed can be rapidly core-cooled in situ by the selective infusion of chilled fluids. At this point the liver can be precooled (body temperature of $30^{\circ}$ to $32^{\circ} \mathrm{C}$ ) by the infusion of cold Ringers lactate solution through the splenic vein cannula prior to aortic crossclamping, or the aorta can be cross-clamped at the diaphragm immediately after cannulation and without liver precooling. Cold preservation solution is then rapidly infused through the aortic and portal cannulae, while the liver is decompressed by dividing the inferior vena cava in the chest. This technique also allows the immediate cooling and protection of the kidneys, 
which are removed once the liver is out. ${ }^{9}$ The heart is cooled separately by its own specific cannulation and solution and excised prior to liver removal. ${ }^{9}$ The choice of solution for infusion is somewhat variable at the present time umong centers. but for livers, we prefer to cool with Ringer's lactate in situ. followed by a final infusion through the portal vein and hepatic artery with the new and much superior Lniversity of Wiscunsin (LWV) solution. once the liver has been completely excised. This new solution developed by Belzer and his associates in Madison. Wisconsin has significantly prolonged the acceptable preservation limits of the liver from the traditional 6 to 8 hours to a remarkable 18 to 24 hours.

After the organs have been cooled in situ. the origin of the hepatic arterial supply is detached from the aorta, the portal vein is divided, and the suprarenal infrahepatic vena cava is transected with part of the right adrenal gland to allow easy identification and ligation of the right adrenal vein at its entry into the retrohepatic inferior vena cava. The liver is removed with a piece of diaphragm containing the suprahepatic vena cava. The liver is flushed at the back table with the CW solution and immediately packaged in a fluid-filled bag that is packed on ice for transport.

The aortoiliac arteries and iliac veins are harvested as grafts and may be required for the reconstruction of the hepatic arterial supply and portal vein in recipients with inadequate native vessels.

The standard technique of liver procurement often requires 2 or more hours of preliminary and meticulous dissection of the hepatic hilum. Such manipulation of the liver could inadvertently produce warm ischemic injury to the liver by interfering intermittently with either portal or hepatic artery flow and could increase the risk of injury to vital structures, especially an anomalous arterial supply to the liver. Furthermore, such a lengthy procedure could not be tolerated in an unstable donor and is not always conducive to collaboration among different transplant teams.

Thus a modification of this technique was developed that was both desirable for and provided a resolution for such circumstances. Although this "rapid" technique requires a much higher degree of skill, this can be achieved unce the "standard" operation has been mastered. Similarly, this technique allows the retrieval of multiple, good quality organs. ${ }^{11}$

The "rapid" technique requires no preliminary dissection except for encirclement of the proximal aorta and cannulation of the inferior mesenteric vein and distal aorta. " Once the thoracic procurement team is ready to arrest the heart or the donor spontaneously arrests, the aorta is crossclamped and rapid. cold infusion is begun through both cannulae. The heart is removed and the liver is allowed to completely flush and cool. The hilar dissection cun then be completed safely in the cold, and rapidly in a bloodless field: at the same time any anomalous vessels are easily identified and preserved. The remainder of the organectomy is carried out as already described for the "standard" technique.

The preparatory steps by the liver and kidney procurement teams require only 15 to 20 minutes. Removal of all the major organs (heart, liver. and kidneys) can be accomplished within 30 to 60 minutes after beginning the donor operation. The "rapid" technique of organ procurement has greatly increased the practicality of harvesting under various adverse 
circumstances and should be applicable in countries without brain death criteria.

The final preparation of the liver graft occurs in an ice basin on a back table in the recipient operating room. Extraneous tissues including the diaphragm and adrenal gland are removed, and all vascular cuffs are prepared for anastomoses. Anomalies are reconstructed at this stage and reported to the recipient surgeon so that strategy can be modified to accommodate the graft.

\section{RECIPIENT HEPATECTOMY}

A bilateral subcostal incision. with an upper midline $\mathrm{T}$ extension and excision of the xiphoid process. usually gives excellent exposure. The exact location of the incision is often dictated by previous right upper quadrant incisions and by the size and configuration of the liver. Excision of the xiphoid process permits easier access to the hepatic veins and suprahepatic vena cava. Additional thoracic extensions are almost never needed. Previous right upper quadrant abdominal operations can render the incision and the obtainment of exposure of the plane of dissection and operative field very demanding and tedious. Bleeding from the wound is sometimes troublesome and it may be necessary to resort to continuous hemostatic sutures at the cut edge of the fascia and peritoneum using polypropylene.

The recipient hepatectomy usually represents the most difficult stage of the entire liver transplant procedure. There is no single best method to remove the diseased native liver. After adequate exposure is obtained, the condition of the liver must be assessed and a plan is taken as to whatever technical approach will be permitted by the abnormal anatomy. It may be impossible to dissect the structures of the hepatic hilum individually because of prior surgery or because of massive formation of varices. Control of the hepatic arterial and portal venous bluod supply may be necessary prior to mobilization of the liver from the hepatic fossa to prevent massive hemorhage. Currently in Pittsburgh, the recipient hepatectom! in almost all adults and in selected pediatric patients is performed using a veno-venous bypass. $^{3.4}$

\section{Veno-venous Bypass}

The most phvsiologically turbulent and critical period of the recipient operation is the anhepatic phase when the native liver has been removed and the inferior vena cava and portal vein are occluded. This obligatory clamping of the splanchnic and systemic venous systems results in a massive sequestration of blood volume in the mesenteric venous circulation and in the peripheral venous circulation of the lower body. The sequelae include diffuse edema of the gastrointestinal tract with prolonged postoperative ileus, renal venous hypertension with postoperative renal dysfunction requiring dialysis. and amplification of the hemorrhage from thin-walled venous collaterals and from any other raw surfaces of the operative wound. Cardiovascular instability requires volume preloading, which can then easily lead to hypervolemia and pulmonary edema after liver revascularization. 


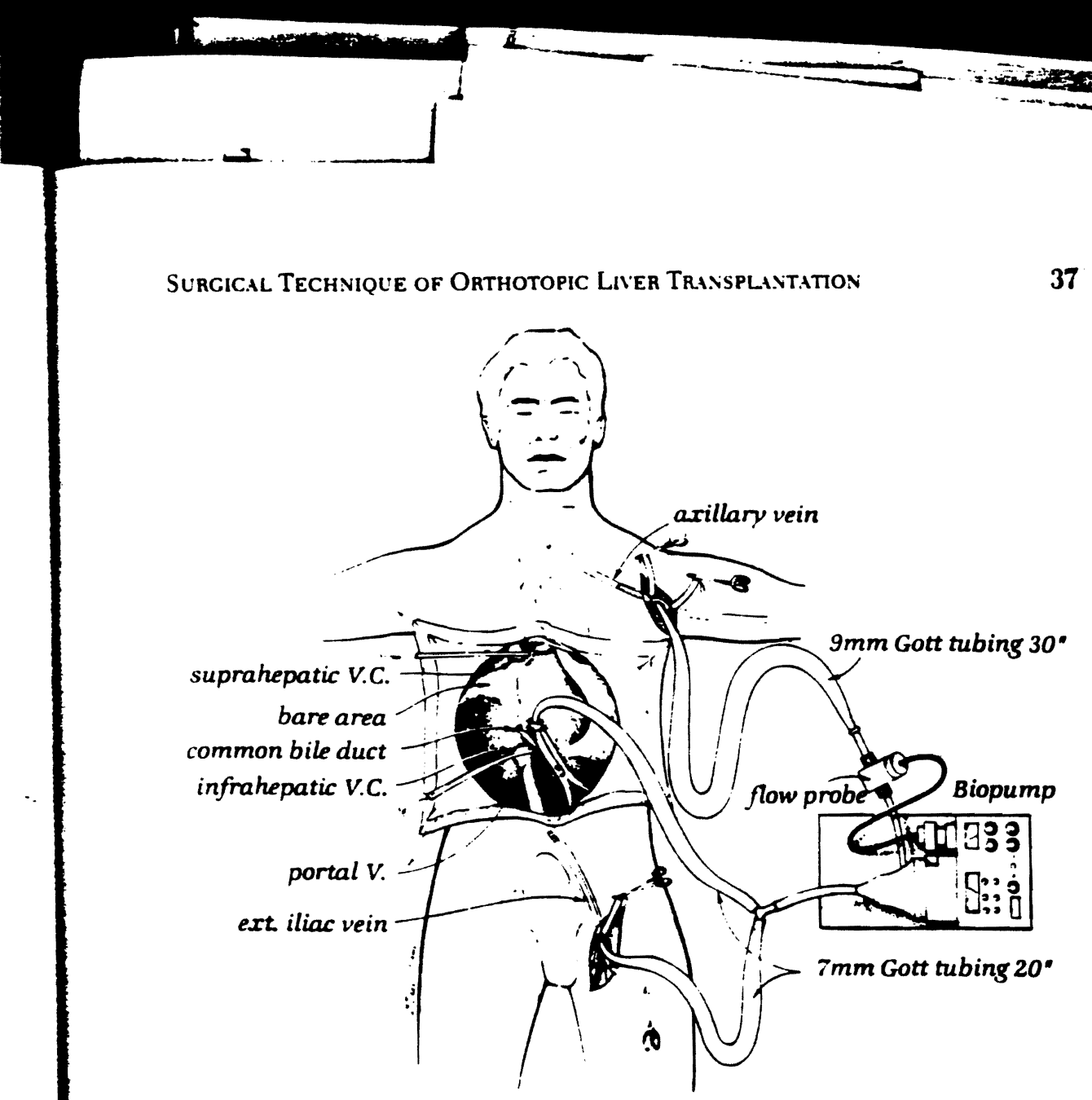

Figure 1. Veno-venous bypass without systemic heparinization during the anhepatic stage of liver transplantation. (Reprinted by permission of Thomas E. Starzl. M.D. Ph.D.. University of Pittsburgh. and Surg Gynecul Obstet 160:270-272. 1985.)

The high potassium and acid load returned to the systemic circulation by the stagnant blood can result in significant instability and cardiac standstill.

The almost routine use of a pump-driven veno-venous bypass system (Fig. 1) without systemic heparinization during the anhepatic phase of the adult recipient operation has resulted in the maintenance of physiological stability and has contributed to a significant reduction in morbidity and mortality. ${ }^{3}$ Hemodynamic parameters can be maintained at prehepatectomy levels using the bypass. The intestinal tract remains uncongested, the incidence of renal failure requiring postoperative dialysis has decreased significantly, and the operative blood loss is reduced significantly because high pressure in venous collaterals is prevented and the large bare areas created by the hepatectomy can be more easily controlled surgically. The use of this bypass system has allowed transplantation in higher risk patients, such as older patients with decreased cardiac reserve and patients with poorly developed collateral vessels, who otherwise might not tolerate venous occlusion.

Because of the stability afforded by the bypass, a longer anhepatic phase is possible, permitting more operative options and numerous modifications in the technique of recipient hepatectumy. Thus the extent of the preliminary dissection can be greatly decreased. especially in patients with severe portal hypertension, previous upper abdominal surgery, or those

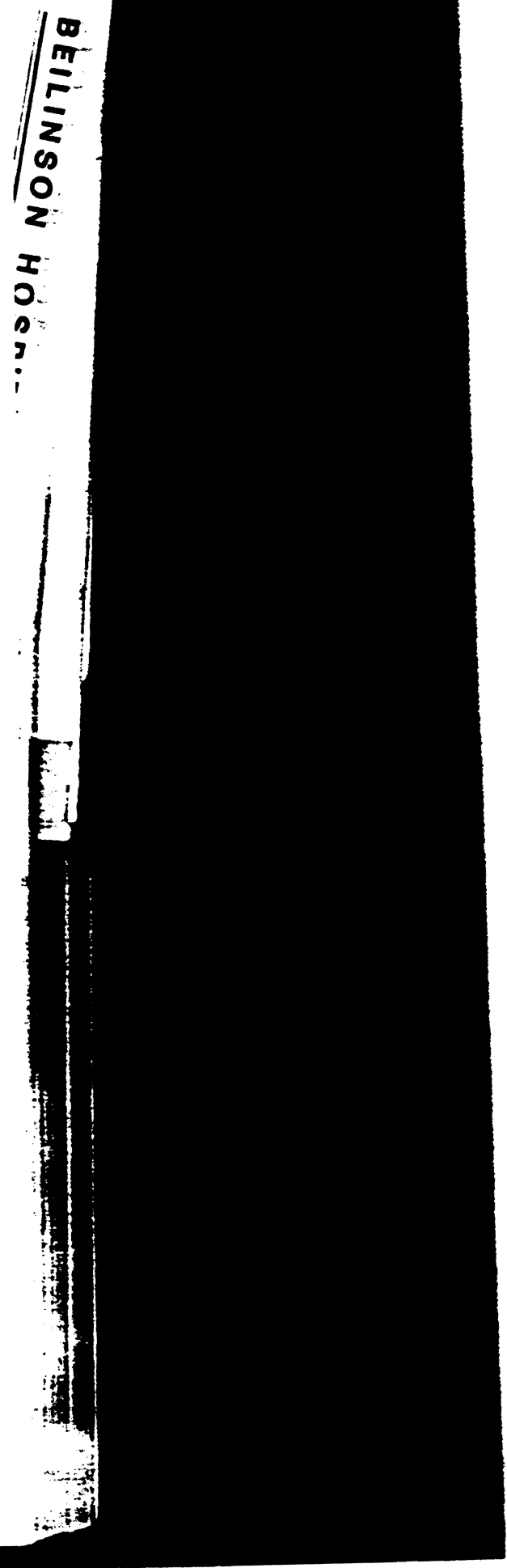


with excessive bleeding. Once the portal triad has been encircled, its three constituent structures can be individually freed with relative safety. Segments of the vessels and bile duct long enough to permit subsequent anastomosis are developed. The hepatic artery and common bile duct are ligated and divided. The left and right branches of the portal vein are ligated and divided, and the portal vein cannula for veno-venous bypass is inserted. A cannula is also introduced into the inferior vena cava by way of the saphenofemoral junction. This allows both the splanchnic and systemic venous systems to be brought into the veno-venous circuit. The bypass circuit is completed by placing a cannula into the superior vena caval system by way of the ipsilateral axillary vein (see Fig. 1). Flow rates in the bypass circuit of 1 to 4 liters of blood per minute are usually possible in adults, and simultaneous occlusion of the portal vein and inferior vena cava cause little change in cardiovascular dynamics. Early placement of venous bypass greatly facilitates mobilization of the liver from the hepatic fossa and especially the dissection of the vena cava. The remaining structures holding the liver. including the falciform, left triangular, and coronary ligaments are divided. The bare areas are entered on both the left and right sides. The right lobe of the liver can then be elevated into the wound, allowing the supra- and infrahepatic segments of the vena cava to be encircled and clamped. The liver can then be shelled out, and the vena caval cuffs for eventual anastomosis can be developed.

After the liver has been removed, the raw surfaces created during the hepatectomy are closed using multiple rows of continuous polypropylene sutures. This is feasible only because of the veno-venous bypass, which allows sufficient time to achieve satisfactory hemostasis and minimize bleeding during the implantation of the new liver. Appropriate vena caval cuffs and sufficient portal vein length must be developed before the graft is brought into the field for anastomosis. This may require ligation and division of the right gastric and gastroduodenal branches of the hepatic artery.

\section{Surgical Options}

In some patients, the presence of severe portal hypertension or adhesions from previous upper abdominal surgery render removal of the diseased host liver using conventional techniques almost impossible. If the hepatic hilum is frozen, or dangerous or difficult to dissect, the entire hilum can be encircled as early as possible and a vascular clamp placed across all hilar structures, which are then transected en masse. The individual structures are then identified, dissected back, the hepatic artery and common bile duct ligated, and the portal vein cannulated for bypass. The remainder of the procedure is as already described. In certain situations, when it is impossible to encircle either the upper or lower vena cava, the liver can be' shelled out from either above or below, by initially transecting either the supra- or infrahepatic vena alone, which represents a much more difficult technique of recipient hepatectomy.

Veno-venous bypass is not used routinely for pediatric liver transplantation in Pittsburgh, because infants and small children tolerate venous occlusion and the anhepatic state reasonably well, certainly much better 

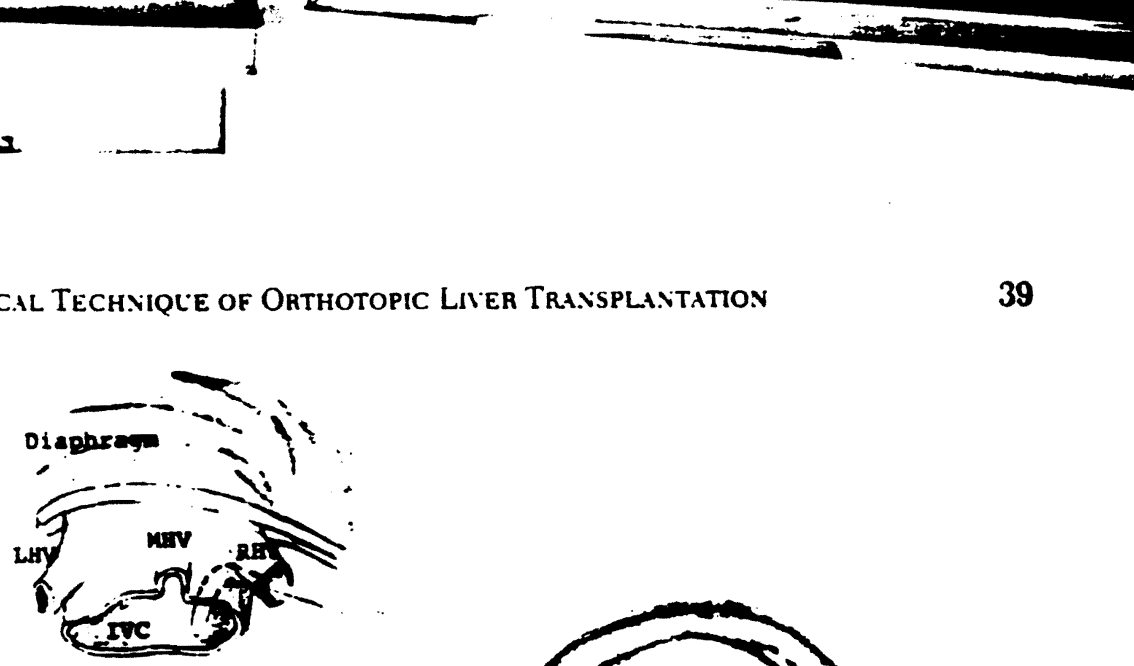

A

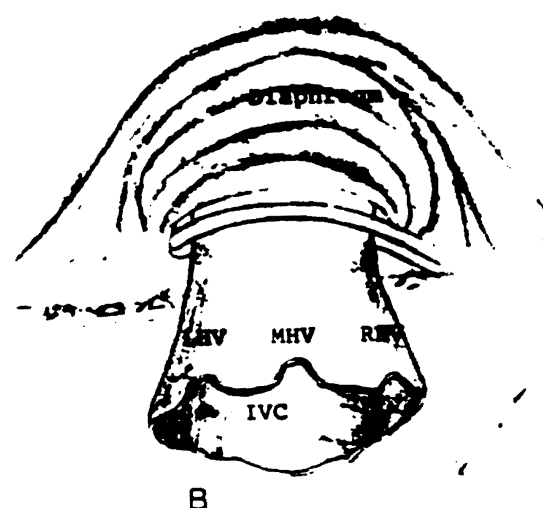

Figure 2. The fashioning of the suprahepatic caval cuff: The stumps of the main suprahepatic veins are opened in continuity with the main lumen. forming a common funnel. i $\mathrm{LHV}=$ left hepatic vein: $\mathbf{M H V}=$ middle hepatic vein: $\mathbf{R H V}=$ right hepatic vein: $\mathbf{I V C}=$ inferior vena cava)

than in adults. However, low-flow pediatric veno-venous bypasses are feasible and useful in selected cases.

\section{GRAFT IMPLANTATION}

\section{Preparation of the Vena Caval Cuffs}

Once hemostasis has been assured, including the oversewing of the retrohepatic "bare area" with continuous sutures if required, the supraand infrahepatic caval cuffs are prepared. The infrahepatic cuff can be very easily fashioned by only trimming of the vena cava that remains following hepatectomy; the search for possible leaks from small tears or avulsed branches must be very thorough. It is much easier to deal with these leaks at this time, rather than after revascularization. For preparation of the suprahepatic caval cuff, the rough cuff that results from the excision of the suprahepatic veins and the upper retrohepatic vena cava must be trimmed and opened by incising the septa between the hepatic veins and the cava (Fig. 2), in such a manner as to obtain a single lumen shaped like a funnel, having a rather generous diameter. Any redundant tissue around the circumference of this cuff is then excised. so as not to have excessive cuff length. Continuous nonabsorbable monofilament polypropylene sutures are used for all vascular anastomoses. 

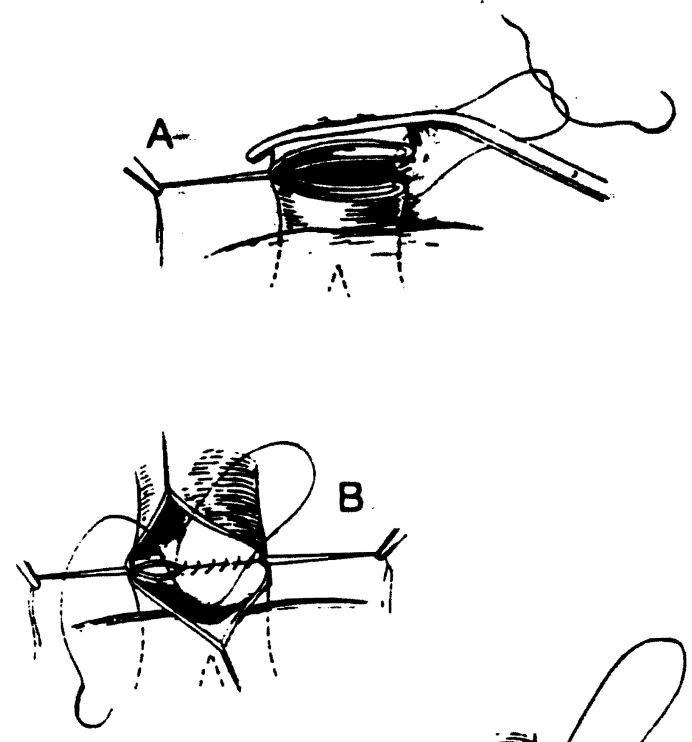

Figure 3. Anastomosis of the suprahepatic vena cava.

\section{Suprahepatic Caval Anastomosis}

At this point two corner sutures are placed in the recipient's suprahepatic cuff, the new liver is brought into the wound, and then the inside needles of the sutures are passed out through the donor liver suprahepatic cuff (Fig. 3A). A third suture can also be placed at this time at the midpoint of the posterior wall, everting the two lips; traction of the corner and middle sutures will form an everted ridge of caval tissue, which will facilitate endothelium-to-endothelium approximation (see Fig. 3). The left corner suture is tied down, while the right corner suture is anchored with an atraumatic clamp and placed under slight tension. The two ends of the left suture are then run to the right, first the everting posterior laver (see Fig. $3 B$ ), then the anterior laver, then tied, with a very small "growth factor" in the right corner (see Fig. 3C). The posterior middle suture, if used. is removed before performing the anterior laver. The right stay suture is then tied down snugly, thus preventing the "runner" with the "growth factor" from unraveling (see Figure 5, portal vein anastomosis illustration, for similar technique and explanation).

\section{Infrahepatic Caval Anastomosis}

Exactly the same technique is then used to anastomose the infrahepatic. vena cava (Fig. 4). During the performance of the anterior wall of this suture line, the liver is flushed with 100 to $250 \mathrm{ml}$ (depending on liver size) 
Figure 4 . Performance of the intrahepatic caval anastomosis. with Hushing of the liver during suture of the anterior wall.

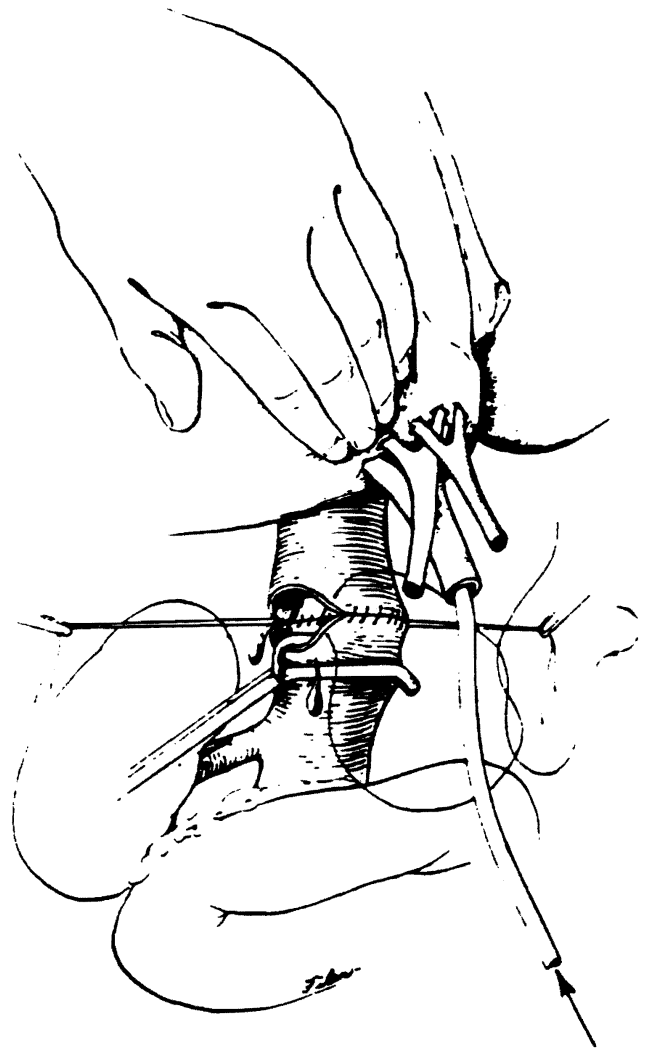

the high-potassium containing preservation solution and the air entrapped inside the liver.

\section{Portal Vein Anastomosis}

The portal cannula (if the veno-venous bypass is used) is then removed and both the recipient and donor portal veins clamped and trimmed to the appropriate length. The utmost care is necessary at this point to ensure that the resulting vessel length is not excessive. since there is a tendencv to err on the side of redundancy. The same everting suture as already described is employed, although the middle stitch is unnecessary: the "growth factor" must be generous, approximately one diameter in length. to prevent an hour-glass type of stenosis (Fig. 5A. B. and C). With this technique. no stricture should result from a straight end-to-end anastomosis. and cutting the two ends at a bias is superfluous.

At this point the portal. infrahepatic, then suprahepatic vascular clamps are removed and the liver is revascularized. After checking for major leaks and controlling anv that are found with interrupted sutures), the venovenous bypass is interrupted and the remaining cannulae removed.

If the portal vein is thrombosed. extremely diseased. or too short las in the cusse of a second or third retransplantation), a free-standing vein graft 

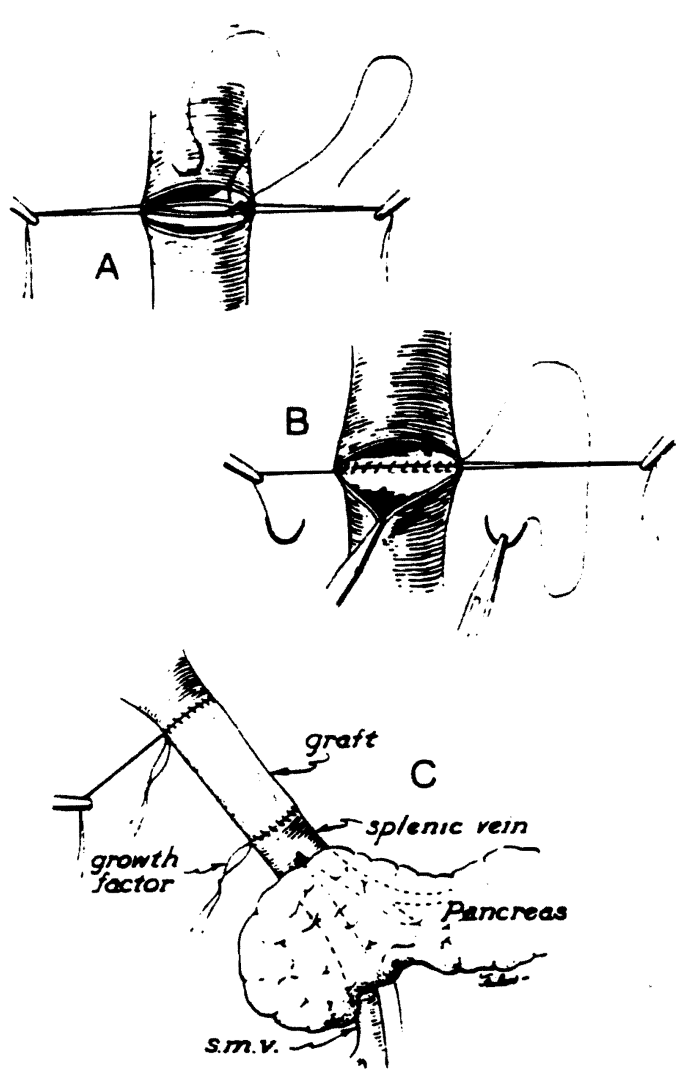

Figure 5. Technique of portal vein anastomosis and of portal vein reconstruction using free-standing donor iliac vein graft. "Growth factor"- the two ends of the running suture are tied together awav from the vessel wall to prevent suture line stenosis.

(with iliac vein removed from the donor) is used to reconstruct or bridge the gap (see Fig. $5 C$ ) usually at or near the confluence of the splenic and superior mesenteric veins (but on occasion directly to the superior mesenteric vein. behind the pancreas, if the splenic vein is completely occluded), flushed with heparinized solution. then clamped. Only a femoroaxillary bypass is used in these cases. omitting the portal cannula and. at the appropriate time. the donor portal vein is anastomosed to the graft. after having adequately trimmed both. "Grouth factors" should be used for both anastomoses (see Fig. 5C).

An "expansion" or "grouth factor" is used to prevent suture line stenosis. especially for the portal vein and hepatic artery. ${ }^{10}$ The running suture is tied several millimeters or more from the wall of the blood vesel. so that when the vessel distends under pressure or when vasospasm resolves, the suture can soak into the vessel. and deformity at the anastomotic line is prevented. A stay suture tied flush to the vessel immediately adjacent to the "growth factor" prevents separation and leakage of the anastomosis at this point. 


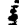

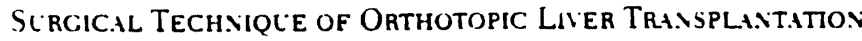

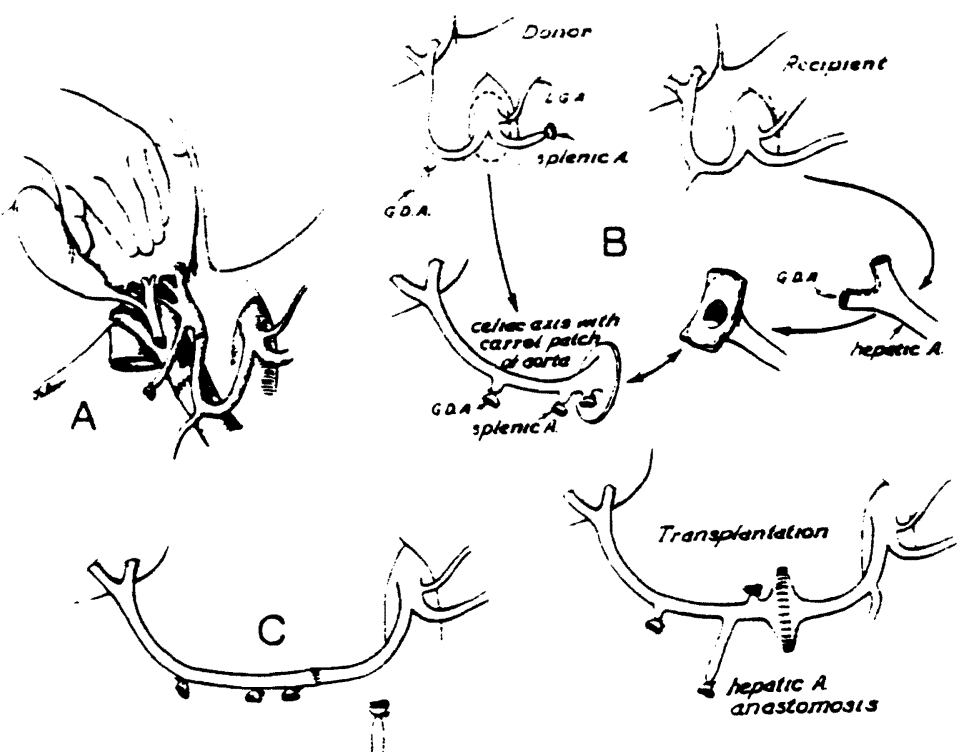

Figure 6. Various methods for primary anastomosis of the hepatic arterv.

\section{Hepatic Artery Anastomosis}

The dissection of the recipient hepatic artery now follows, if this was not done during the hepatectomy. It is carried as proximally as necessary to obtain a vessel of good caliber and optimal blood inflow (usually 1 to $\dot{2}$ $\mathrm{cm}$ proximal to the take-off of the gastroduodenal branch). An end-to-end anastomosis is then performed between the donor and recipient arteries. We generally prefer the same type of running suture, with a "growth factor" of variable length depending on the local conditions, that is utilized for the veins. The level of the anastomosis on the donor and recipient side depends on the original length and diameter of the vessels, as well as other circumstances that vary from patient to patient. We usually prefer to anastomose the donor celiac axis to the recipient vessel at the level of the proper hepatic artery (Fig. 6A), at the gastroduodenal artery bifurcation (see Fig. 6B), which forms a "pseudo" Carrel patch, at the level of the common hepatic artery (see Fig. 6C), or even at the level of the celiac axis after having ligated the splenic and left gastric arteries. It is important to assess the length of the resulting vessel very carefully: it should be either short, barely sufficient, or significantly redundant, forming a "lazy" loop, so as not to suffer kinking, which leads to thrombosis. An artery that is only slightly too long will actually have a much greater chance of kinking; therefore, this situation must be avoided.

When an aberrant right hepatic artery originating from the superior mesenteric artery is present. there are two basic methods of reconstructing the vessels at the back-table, with the goal of producing a single-donor channel for subsequent anastomosis to the recipient hepatic artery. ${ }^{2}$ : Either the "fold-over" technique ${ }^{2}$ (Fig. $i A$ ) or the end-to-end anastomosis of the right branch to the stump of the splenic artery: (see Fig. $i B$ ) can be 


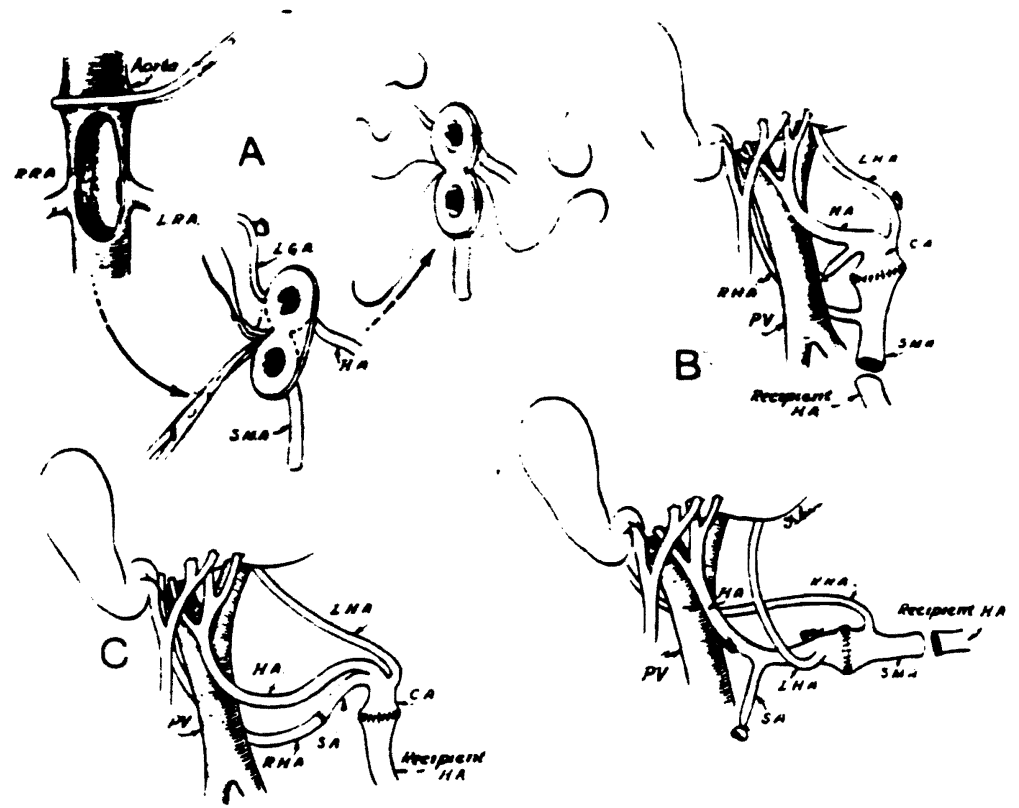

Figure $i$. Reconstruction of the donor hepatic arten when a right branch from the superior mesenteric artery is present.

employed. Lately, we have favored the right hepatic/splenic design, because it appears to be easier to position at the completion of the rearterialization of the liver.

If a direct anastomosis to the recipient hepatic artery is not possible or indicated (because of gross size mismatch, poor blood inflow, intimal dissection. or insufficient length), an arterial graft must be emploved. In the adult and in a majority of pediatric cases. a free-standing graft of donor iliac artery is used to arterialize the liver from the infrarenal aorta. and is passed in front of the left renal vein and behind the pancreas (Fig. $8 \mathrm{~A}$ and $B$ ). Four tunnel routes ("long", two "medium," and "short") can be used. and the choice will depend on the local conditions, length of the graft. and
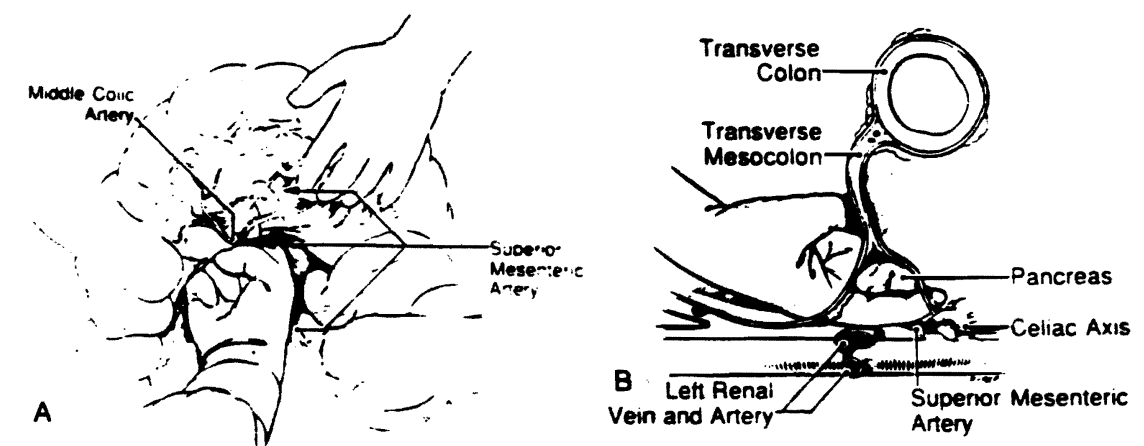

Figure 8. A. Performance of the retropancreatic tunnel for placement of an aortohepatic arterial graft. B. Cross-sectional view. 

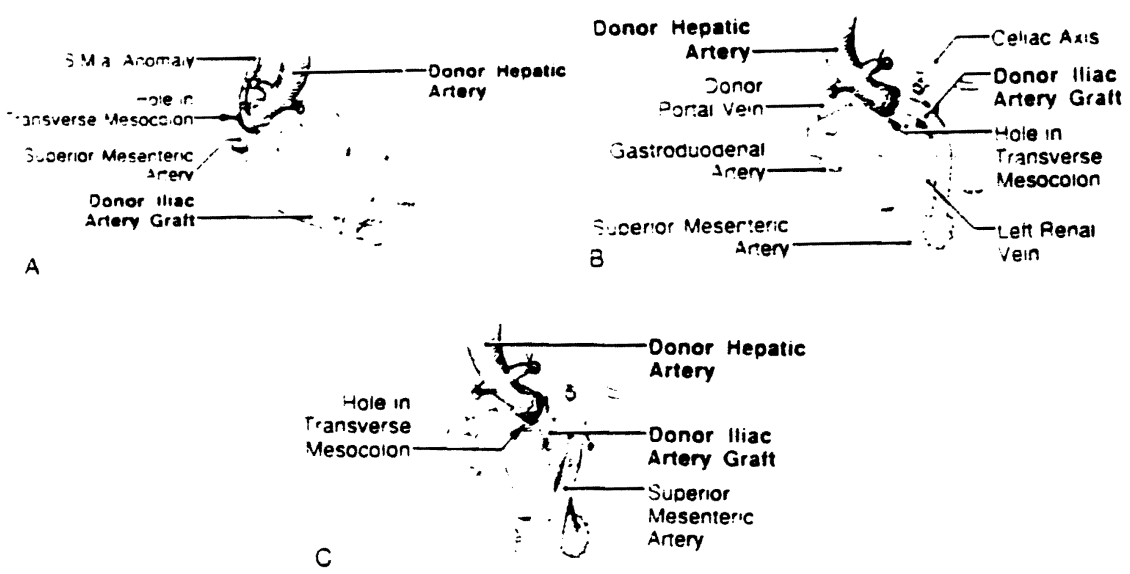

Figure 9 . The various tunnel routes for the aortohepatic graft. A. Long tunnel: $B$, short tunnel: $C$. inedium tunnel.

preference of the surgeon (Fig. $9 A, B$, and $C$ ). We never use synthetic material for this graft. since the risk of infection in the immunosuppressed transplant patient is far too great. If a vascular graft from the same donor is not available, we use a vessel of the same blood type, harvested from other recent donors (within 5 days), and kept refrigerated in the operating room.

In the very small pediatric patient. the arterial anastomosis can be performed by using the donor aorta, which is harvested in continuity with the liver, the distal end of which is then anastomosed to the recipient infrarenal aorta as a "conduit" (Fig. 10). All the collateral branches (renal, lumbar, adrenal. phrenic, and superior mesenteric. if there is no aberrant right hepatic branch) are ligated before implantation, while the supraceliac end is transected on a bias and closed with a double running suture. Occasionally, if the abdominal aorta cannot be used, the entire descending thoracic aorta is removed, transected just above the celiac axis, and then reanastomosed on the inferior aspect, to form an antegrade "conduit"; the supraceliac aortic end is closed with a double running suture. The use of aortic conduits should only be reserved for very small children, or in other rare situations when it is impossible to use an iliac artery graft.

All vascular anastomoses must be performed with meticulous detail, particularly, successful reconstruction of the portal venous and hepatic arterial circulations that demand flawless technique. Failure of either anastomosis usually leads to patient death or retransplantation. If there is any doubt as to the vessel length or blood flow, the anastomosis should be redone, and vascular grafts used, if necessary.

\section{BILIARY TRACT RECONSTRUCTION}

Having completed the rigorous task of hepatic revascularization, attention is then focused on the establishment of biliary tract continuity. The 


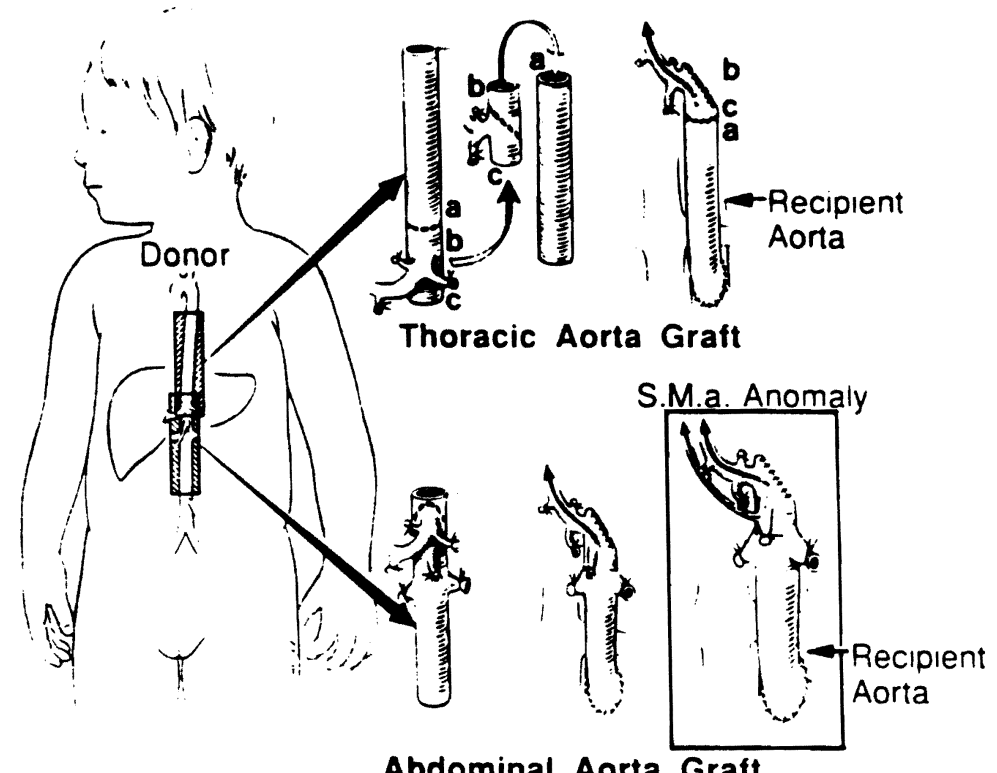

Figure 10. The techniques of aortic "conduits" in pediatric recipients.

success of the entire liver transplant procedure depends upon the biliar. tract reconstruction. and the same degree of meticulous attention and concentration already used is still necessary. Complete hemostasis should be achieved before embarking on the biliary tract reconstruction. because bleeding is much more difficult to control afterward.

Biliary tract leaks or obstructions are the most frequent technical complications following liver transplantation. ${ }^{4}$ Fortunately. most of these. if recognized and dealt with promptly. can be successfully managed. Standardization of our methods for biliary tract reconstruction has significantly reduced the incidence of these biliary tract complications.

Duct-to-duct reconstruction over an external T-tube stent is our preferred method of reconstruction in patients without preexisting extrahepatic biliary tract disease and when there is no significant size discrepancy. between the donor and recipient bile ducts. Advantages of this method include preservation of the sphincter of Oddi and availability of the T-tube to monitor bile production. and to perform cholangiography. The T-tube provides an important mold for the healing bile duct. and the upper limb of the T-tube should reach but not obstruct the hepatic duct bifurcation. The T-tube is usually left in place for about 6 to 8 weeks.

In many patients, preexisting disease of the extrahepatic biliary system or unfavorable anatom! precludes direct duct-to-duct repair. Our alternative preferred method of reconstruction under such circumstances is a Rouxen-I choledochojejunostomy over an internal stent. The main indications for this procedure include recipient biliary tract disease ie.g. sclerosing cholangitis. liver tumors where an extensive supraduodenal lymph node dissection includes the recipient bile duct, size discrepancy between the 
donor and recipient ducts, questionable blood supply to the recipient duct, and spasm of the sphincter of Oddi). Anastomosis of the donor bile duct to the side of the distal portion of an 18 inch Roux-en-Y limb of proximal jejunum is used. The duct anastomosis is performed over a small polvethy lene pediatric feeding tube catheter that eventually passes out spontaneuusly through the gastrointestinal tract. Roux-en-Y choledochojejunostomv is the safest method of biliary reconstruction. with a complication rate of less than 8 per cent. Failures of duct-to-duct repair are usually best managed by conversion to this method of reconstruction.

An intraoperative cholangiogram (T-tube or cystic duct cholangiocatheter) is always attempted to check the position of the tube. the patency and integrity of the anastomosis. the anatomy of the biliary tree. and observe free flow of dve into the bowel. Anv problems warrant a redo of the reconstruction.

\section{SPECIAL CONSIDERATIONS FOR PEDIATRIC LIVER TRANSPLANTATION}

\section{Donor Operation}

The pediatric donor operation is fundamentally the same as in adults. The "rapid technique" of multiple organ harvesting offers particular advantages in the pediatric patient. Intraoperative dissection of the liver and kidneys is kept to a minimum and is reserved for the back bench, where conditions are more favorable for meticulous dissection of the fine hilar and vascular structures. Intraabdominal exposure time is considerably reduced. minimizing hypothermic complications and simplifying fluid balance problems resulting from insensible losses. The risk of hepatic congestion from intravenous fluid overload is much less with a shorter operating time.

Several modifications mav be useful in the pediatric donor operation. Proportionately smaller amounts of perfusate solutions are infused through the aortic and portal cannulas. Perfusate overload may cause severe hepatic congestion and possibly result in delayed and impaired function of the new liver in the recipient. It may be difficult or impossible to palpate an anomalous right hepatic arterial branch behind the portal vein in small pediatric donors. Therefore the entire extent of the proximal superior mesenteric artery should be visualized from its origin at the aorta, searching for an anomalous right hepatic branch where it originates from the right side of the superior mesenteric artery. If any doubt exists as to the significance of any small branches seen in that area, then it is best to proceed as if a right hepatic branch were indeed present by removing a Carrel patch of the origins of the celiac and superior mesenteric arteries in the usual fashion. More extensive dissection and examination of the arterial anatomy can then be accomplished at the back bench. The origin of the right renal artery may be dangerously close and even superior to the origin of the superior mesenteric artery in small pediatric donors. so extreme care is required in removing the Carrel patch. If the kidneys are not to be harvested in very small pediatric donors, then the entire abdominal aorta 
may be removed en bloc with the liver for later back bench preparation for the recipient.

The most serious problem facing the pediatric liver transplant candidate is the scarcity of small donors. Despite improved organ donation rates and liberalization of organ donor criteria. the demand still far outweighs the supply of very small pediatric livers. One possible solution to this problem is segmental liver transplantation of a portion from the more often available adult liver. Surgical reduction of the larger intact liver is performed on the back bench. typically sparing the left lateral segment or the left hepatic lobe for recipient grafting. In Brussels, Otte and colleagues have reported the largest series to date with an 80 per cent success rate in 15 patients with no increase in postoperative complications and no adverse effects on liver function. Success rates in other smaller series have been about 30 per cent. Further studies will determine the feasibility of this option.

\section{Recipient Operation}

The recipient hepatectomy may be technically somewhat easier in children than in adults, unless there have been multiple previous abdominal operations. such as Kasai portoenterostomies. Blood loss may be lower because of less extensive venous collaterals from portal hypertension than in adult cirrhotic patients. The costal margins are quite mobile and the abdominal wall flexible enough so that an upper transverse incision incorporating a previous incision. if present, provides excellent exposure in most cases.

One of the most significant technical improvements in pediatric liver transplantation has been the standardization of the bile duct reconstruction. In the early experience, a variety of biliary reconstructive procedures were employed. most commonly, cholecystoduodenostomy with ligation of the common duct. The inordinately high complication rate ( 35 per cent) was manifested by bile duct obstruction and fistula. Bacteremia was also common even with a technically satisfactory reconstruction. probably as a result of constant contamination of the biliary tract from the duodenum; cholecystoduodenostomy was therefore abandoned. The preferred method of biliary tract reconstruction now is like in adults. a duct-to-duct anastomosis over a T-tube. when possible. Unfortunately this is not an option in more than three out of four pediatric recipients because the common duct is either absent (as in biliary atresia) or too small for satisfactory anastomosis. In such cases, an end-to-side choledochojejunostomy to a Roux-en-Y limb over an internal stent is the preferred method. Complications with the current methods of biliary reconstruction have been less than 10 per cent. The previously constructed Roux-en-Y limb in patients with biliary atresia who have had Kasai portoenterostomy may be used again for biliary reconstruction at the time of liver transplantation as long as the intestine is not damaged during the recipient hepatectomy dissection. The presence of an ostomy or previous closure of a stoma may result in a functional length of Roux limb that is not long enough to reach the common duct without placing undue tension on the biliary anastomosis. In such cases, the existing Roux-en-Y limb is taken down and a new Roux limb is constructed.

Multiple previous operations for Kasai portoenterostomies. revisions. 
and stomal manipulations mav cause extensive adhesions around the hilum often resulting in a "frozen" upper abdomen incorporating the stomach. duodenum. transverse colon, multiple loops of small bowel, and the hilum. Dissection of the existing Roux limb may be impossible, and construction of a new Roux limb may be quite difficult. Safe dissection of the hepatic artery and portal vein embedded deep within the hilum may be jeopardized. In such cases. the hepatectomy may otten be performed more easily and with greater safety by dissecting the hilum from a retrohepatic superior approach after first crossclamping and dividing the suprahepatic inferior vena cava. From this approach. the vascular structures of the hilum will be encountered initially in an operative field less involved with adhesions, reserving dissection of the Roux limb and portoenterostomy until just prior to removal of the diseased liver. Such a maneuver will often result in considerably less blood loss and in an appreciably greater saving of time in completing the recipient hepatectomy

The introduction of the veno-venous by pass technique has dramatically altered the intraoperative management of the anhepatic phase of the liver transplant operation in adults. This technical advance was not initially made available to pediatric recipients because of concern that bypass flow rates of less than $1000 \mathrm{ml} /$ minute would lead to clot formation within the tubing and subsequent iatrogenic pulmonary embolism. However, recent studies have demonstrated that the veno-venous bypass svstem can operate safely with much lower flow rates. Therefore, it is current practice to utilize the bypass for all children over $15 \mathrm{~kg}$.

\section{HEPATIC RETRANSPLANTATION}

Hepatic retransplantation may be required for any of the following reasons: (1) primary graft nonfunction: (2) rejection; (3) technical failure (i.e., hepatic artery or portal vein thrombosis); ( 4 ) recurrent disease. Once hepatic failure occurs, retransplantation should be performed expeditiously prior to the development of multiorgan failure or the onset of sepsis.

Depending on the timing and the cause of the retransplantation, varving degrees of difficulty may be encountered during the hepatectomy. As the time interval since the previous transplant increases, the more likely it is that dense adhesions will be encountered. Early placement on venovenous bypass may facilitate the hepatectomy in such cases.

Although most of the steps in performing a retransplant are similar to those of a primary transplant, several important concepts must be considered in the planning of these procedures. The importance of donor vascular grafts and their various uses has been described above. Alternate methods of arterial or portal venous reconstruction are often required at retransplants. Thus the harvesting of good lengths and quality of venous and arterial grafts is critical.

It is usually preferable to excise all previous anastomoses before inplanting the new gratt, if adequate recipient cutfs can be developed. However, this is not always possible, especially for the upper vena cava. Extra length may be obtained in preparing the upper caval cuff by leaving 
the original anastomosis intact and seuing the new donor cava to a short cuff of vena caly from the first liver. This extra length provided by the cuff of vein may prove to be life-saving. even if the new sutures encompass the original anastomosis.

Special attention must be paid to the hepatic arterial reconstruction. Apart from previously placed iliac artery grafts on the infrarenal aorta, which can be reused. all other arterial anastomoses should be excised.

Although the recipient bile duct can be reused for a duct-to-duct anastomosis under certain specific circumstances. it is usually necessary and preferable to use a Roux-en-Y choledochojejunostomy. If the jejunal limb is to be reused. the original site of the duct anastomosis should be excised or closed primarily. and the anastomosis should be performed at a new site along the jejunal limb.

The results of retransplantation are in a large part dependent on the timing and setting. Retransplantation occurs under three settings: emergent. acute, and elective. Emergent transplantation is required for profound hepatic failure, resulting from aggressive acute rejection. arterial thrombosis, or primary nonfunction and is dependent upon an effective organ procurement system and optimal use of all available donors. Rejection is primarily responsible for the need for retransplantation in the acute and elective settings. and for this reason. usually carries the best results.

\section{SUMMARY}

Although significant strides have been made in the surgical technique of orthotopic liver transplantation, numerous problems and nuisances are still encountered. Further surgical refinements will certainly evolve. The development of better preservation techniques. the use of intraoperative flowmeters, and the availability of new technologies. such as an artificial liver. should impact and advance the techniques of liver transplantation significantly and improve the overall results even further.

\section{REFERENCES}

1. Esquivel CO, Iwatsuki S. Gordon RD, et al: Indications for pediatric liver transplantation. J Pediatrics, in press

2. Gordon RD. Shaw BWV Jr. Iwatsuki S. et al: A simplified technique for revascularization of liver homografts with a variant right hepatic artery from the superior mesenteric arten: Surg Ginecol Obstet 160:474-476, 1985

3. Griffith BP. Shaw BW Jr. Hardesty RL. et al: Veno-venous bypass without systemic anticoagulation for transplantation of the human liver. Surg Givecol Obstet 160:270273. 1955

4. Lerut J. Gordon RD. Iwatsuki S, et al: Biliary tract complications in human orthotopic liver transplantation. Transplantation $43: 4 \bar{i}-\bar{j} 1$. $198 \overline{7}$

5. Shau BII Jr. Gordon RD. Iwatsuki S. et al: Retransplantation of the liver. Semin Liver Dis 5:394-401. 1955

6. Shaw BW Jr. Inatsuki S. Bron $K$. et al: Portal vein grafts in hepatic transplantation. Surg Grnecol Obstet 161:66-68. 1983

7. Shaw BW Jr. Iwatsuki S. Starzl TE: Alternative methods of arterialization of the hepatic graft. Surg Ginecol Obstet 159:490-493. 1984 


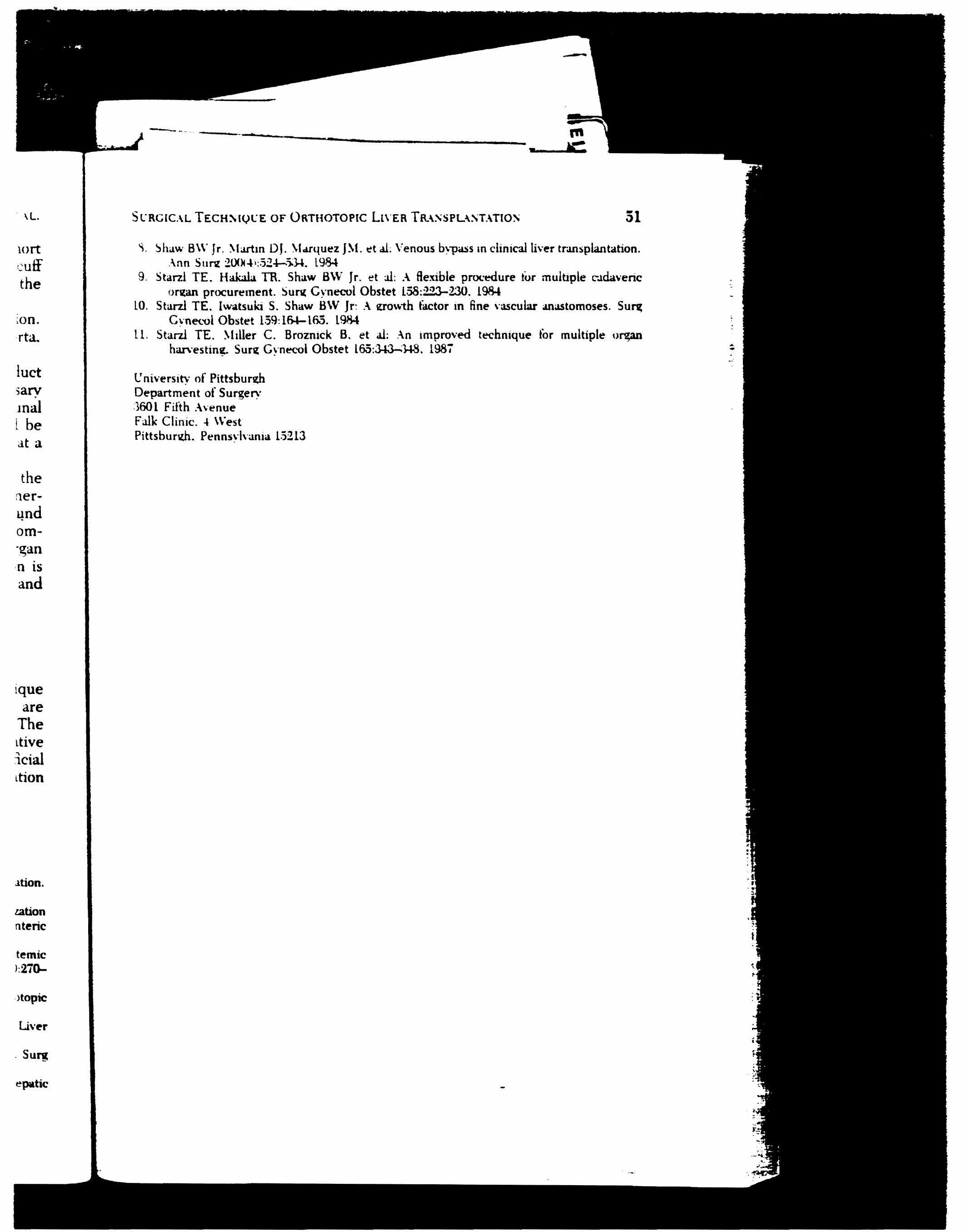

